\title{
Bacteriological Profile and Antibacterial Sensitivity Patterns of Isolates among Burn Patients in Sulaimani City
}

\author{
Kamal Jalal Rashid \\ Anesthesiology Department, \\ Technical College of Health, \\ Sulaimani Polytechnic University \\ Sulaimani, Iraq \\ kamalashty73@yahoo.com
}

\author{
Muhammed Babakir-Mina \\ Medical Lab. Department, \\ Technical College of Health, \\ Sulaimani Polytechnic University \\ Sulaimani, Iraq \\ m.babakir@yahoo.com
}

\author{
Dana Abdilmajid Abdilkarim \\ Community Health Department, \\ Technical College of Health, \\ Sulaimani Polytechnic University \\ Sulaimani, Iraq \\ dana_dr77@yahoo.com
}

\author{
Bestun Ibrahim Hama Rahim \\ Community Health Department, \\ Sulaimani, Technical Institute, \\ Sulaimani Polytechnic University, \\ Sulaimani, Iraq \\ bestunarambestun@gmail.com
}

\author{
Sarko Masood Mohammed \\ Community Health Department, \\ Technical College of Health, \\ Sulaimani Polytechnic University, \\ Sulaimani, Iraq \\ $\underline{\text { sarko baban@yahoo.com }}$
}

Abstract: Nosocomial infections have increasingly been implicated in transferring fatal septic complications in burn patients. Also multidrug resistant profiles of microorganisms are being increasingly found in burn wounds which are very much alarming due to the limited number effective antibacterial drugs. Retrospective data were collected from burn patients at Sulaimani Burn and Plastic Surgery Hospital between January 2013 and December 2015. Culture\& sensitivity tests were performed using wound surface swabs and tissue culture over the three year period. Their results were collected in a predesigned digital form. Statistical analysis was done and results plotted. Out of 500 burn positive swabs samples were previously taken from hospitalized patients, the commonest bacterial isolate were grampositive bacterial infection; Methicillin-Resistant Staphylococcus Aureus (MRSA) 215(43\%) followed by gram-negative bacterial infection; Acinetobacter baumannii and Pseudomonas aeruginosa 95(19\%) and 85 (17\%). Vancomycin has no any resistant rate for all gram-positive bacteria followed, but Teicoplanin is the second best drug especially, for MRSA $8.4 \%$. Imipenem and Meropenem are antibiotics with no or less resistant rates for most of gram negative bacteria. Resistance to antibiotics is rapidly increasing in our community and burn wounds are frequently infected by these multidrug resistant organisms. Careful antibiotic selection and effective control of these strains can be translated into lower morbidity and mortality for these patients.

Keywords: Burn patients; MRSA; Antibacterial drug; Multidrug resistant, and Burn Patients

\section{INTRODUCTION}

Infection in burn wound is still considered as the most important cause of disability and mortality in all ages and in both developed and developing countries.1,2 Despite considerable advances in the overall management of burn injuries, infection and the resultant sepsis continues to be a formidable foe for burn care providers. Approximately 50-75\% of mortality amongst burn patients after the initial resuscitation phase, is attributable to various infectious complications. [1]

Burn injury patients are at high risk of infections for a variety of reasons. For instance, the readily available exposed body surface, immunocompromizing effects of burns, invasive diagnostic and therapeutic procedures and prolonged hospital stay. Patient factors such as age, extent of injury, and depth of burns coupled with microbial factors such as the type and number, enzyme/toxin production and motility of organisms are the determinants of invasive infection. Superficial bacterial contamination of the wound can easily advance to invasive infection in these patients. The degree of bacterial wound contamination has a direct correlation with the risk of sepsis [2].

Factors that are associated with improved outcome of burn injury patients and prevention of infections among them predominantly include early excisions and grafting of deep burns together with aggressive infection-control measures. These changes potentially lead to the emergence of antibiotics resistant isolates and treatment failures [3].

Some reports suggest that burn wound infection is the most common type of infection, whereas other reports show predominance of pneumonia and primary blood stream infection [4]. Burn wound infections can be caused by bacteria, fungi, or viruses. Historically, group a Beta-hemolytic Streptococcus was the most frequent cause of life-threatening burn wound and systemic infections. The use of penicillin altered the spectrum of gram-positive pathogens, leading to the emergence of Staphylococcus aureus (S. aureus) as the most common gram-positive early colonizer of the burn wound. In burns, $S$. aureus has been a major cause of morbidity and death. The disruption of the normal skin barrier and the immunocompromised state makes burns an easy target for colonization. Additionally, prolonged hospitalization and antibiotic therapy are risk factors for the development of methicillin-resistant Staphylococcus aureus (MRSA) colonization and infection. [5]

Some strains of Pseudomonas aeruginosa ( $P$. aeruginosa) and Acinetobacter now encountered in burn units are resistant to all the aforementioned antibiotic classes. Over the last 20 years, an increase in resistance of $P$. aeruginosa to reserve antibiotics such as ceftazidime, and a dramatic decrease in susceptibility of Acinetobacter spp. to ceftazidime and ciprofloxacin, has 
developed. Meropenem has been identified as an important reserve antibiotic to which most $P$. aeruginosa and Acinetobacter are susceptible, but resistance to the carbapenems has developed. A study at eight centers in the United States found that $15 \%$ of $P$. aeruginosa isolates recovered from burn ICUs were resistant to ceftazidime and $20 \%$ were resistant to imipenem. Pseudomonas isolates with resistance to ciprofloxacin also have been reported. Outbreaks of $P$. aeruginosa resistant to most available beta-lactams, aminoglycosides, and fluoroquinolones have been reported in burn units, neurosurgical ICUs, and cancer centers and among patients with cystic fibrosis [5].

Due to unavailability of an appropriate alternative antibiotic for hospitalized patients, it may be lifethreatening in some patients. So the present study was conducted to determine the microbial profile of burn patients, the antimicrobial susceptibility patterns of the cultured microbes and the frequency of infections with respect to the duration of burn, among our burn injury patients.

\section{LITERATURE REVIEW}

Antibacterial susceptibility patterns for microorganisms isolated from the hospitalized patients with infectious diseases are continuously evolving. Determining the sensitivity of isolated bacteria to antibiotics helps clinician to use appropriate antibiotics [1].

Also strict infection control practices and appropriate empirical antimicrobial therapy are essential to help reduce the incidence of infections due to these antibiotic resistant organisms [2].

Although burn wound surfaces are sterile immediately following thermal injury, these wounds eventually become colonized with microorganisms. Microorganisms transmitted from the hospital environment become more resistant to those antimicrobial agents than generated from the patient's normal flora $[2,3]$.

Resistance to antimicrobial agents is an increasing clinical problem and is a recognized public health threating. Antibiotic-resistant organisms such as MRSA, Vancomycin-resistant enterococci, and multiple-resistant gram-negative rods, including $P$. aeruginosa, Acinetobacter species, and various members of the family Enterobacteriaceae, have been associated with infections of the burn wound and other anatomic sites in patients with major thermal injury, occasionally in the form of Nosocomial Infection (NI) [4].

Multi-Drug Resistance (MDR) in the Gram-negative isolates was defined as resistance to three or more first line classes (beta lactams, aminoglycoside, and fluroquinolone) of antimicrobials and are usually resistance to $\geq 3$ drugs (third/fourth generation Cephalosporins, Piperacillin/tazobactam, Imipenem, Meropenem, Fluoroquinolones, Aminoglycosides). Although, MDR in the Gram-positive isolates is resistance to three or more first line classes (Beta lactams, Aminoglycoside, and Fluroquinolone) of antimicrobials and are usually resistance to $\geq 3$ drugs (third/fourth generation penicillin, Ampicillin,
Ampicillin-sulbactam, Oxacillin, Ticarcillin/Clavulanic Acid, Cephalothin, Cefazolin, Cefotetan, Cefuroxime, Cefotaxime, Ceftazidime, Chloramphenicol, Erythromycin, Ciprofloxacin, Nitrofurantoin, Gentamicin, Amikacin, Clindamycin, and Vancomycin [5].

A study conducted in North Iran showed that Pseudomonas species have less resistant to antimicrobials like Amikacin (50\%), Gentamicin (42.85\%), Ciprofloxacin (94.4\%), Carbenicillin $(42.85 \%)$, Tobramycin $(87.52 \%)$ and Ceftazidime $(33.3 \%)$, while $S$. aureus were highly resistant to Ampicillin (100\%), Erythromycin (100\%), and Ciprofloxacin (100\%), but Acenetobacter species are highly resistant to all antibiotics with $100 \%$, while for Klebsella species more drug resistant are ceftriaxone, Cefixime, Ciprofloxacin, and Co-Trimoxazole in percent $100 \%$. The same study showed that Enterobacter spp. resistant to most antibiotics such as Ciprofloxacin, Gentamicin, Co-Trimoxazol, and Tetracycline in percent $100 \%$, while E. coli it has similarity to more resistant to antibiotic for the most drugs $100 \%$ [6].

Another study conducted in Iraq indicated that Pseudomonas species is the most MDR for Amoxicillin, Augmentin, Cefadroxil, and Cefotaxime 100\%, followed by Klebsella species about $100 \%$ MDR for Amoxicillin, Augmentin, Cefadroxil, Cefotaxime, and CoTrimoxazole. In addition $S$. arueus was less resistant to previous antibiotic ranged (39.7- 61.9\%) [7]. A Romanian study showed that $P$. aeruginosa, E. coli and Klepsella species were highly sensitive to Colistin about $100 \%$, [8]. In addition, recent published article by Babakir-Mina, M. and his co-workers in Sulaimani, Iraq indicated that Acenetobacter baumannii has no resistant rate to Colistin, followed by Ampicillin/sulbactam (SAM) $2.4 \%$ and Imipenem 5.3\% [9].

\section{METHODS AND MATERIALS}

\subsection{Data management and statistical analysis:}

Retrospective analytic study was conducted in the Sulaimani Plastic and Burn Hospital. The analysis is based on data collected from clinical laboratory examination records of 500 positive cultures from January 2014 to December 2016. The clinical samples were taken for microbiological tests from various body sources (blood, urine, burn wounds) at different time intervals during the patients' stay in hospital. Culture and sensitivity tests were undertaken at the center's microbiology laboratory inside the hospital.

The data were analyzed through SPSS software (Statistical package for social sciences, Version 22). Various descriptive statistics were used to calculate frequencies, percentages, means and confidence interval.

\subsection{Microbiological investigation and antibiotic sensitivity test:}

The swabs were dipped in Stuarts transport medium, and then inoculated on selective and differential medium, enrich medium (blood agar). The isolates were identified using conventional identification techniques after incubation for $18-48$ hours at $37^{\circ} \mathrm{C}$ [10]. Positive cultures were sub cultured on blood agar and 
MacConkey agar, as per routine bacteriological guidelines. API (Analytical Profile Index) 20E system was used to identify the isolated gram-negative bacteria. While gram stains, catalase test, hemolysis on blood agar, coagulase and other tests were used to identify gram-positive bacteria submitted to identification tests. Als latex agglutination test were used as a confirmation stages of investiation. (BioMerieux SA,Lyon, France). [11].

Small filter paper disks $(6 \mathrm{~mm})$ impregnated with a standard amount of antimicrobials were placed onto an agar plate to which bacteria have been swabbed by a bacterial suspension using distilled water comparable to $0.5 \mathrm{McFarland}$ turbidity standard. After 15 minutes of inoculation, the antimicrobial containing discs are applied to the agar with sterile forceps pressed firmly to ensure contact with the agar and then the plates of Muller Hinton Agar were incubated overnight, and the zone of inhibition of bacterial growth was measured for Gram-positive and Gram-negative bacteria. Then the result will be calculated as describe elsewhere [12].

The most common antibiotic discs according of the (Fluka company) used for Gram-positive isolates were: Amoxicillin/Clavulanic Acid (AMC) 20/10mcg, CoTrimoxazole (COT) 1.25/23.75 mcg, Gentamicin (GEN) $10 \mathrm{mcg}$, Vancomycin (VA) $30 \mathrm{mcg}$, Amikacin (AK) 30 mcg, Teicoplanin (TEN) $30 \mathrm{mcg}$, Nitrofurantoin (NET) $300 \mathrm{mcg}$, and Doxycycline (DO) $30 \mathrm{mcg}$. The antibiotic discs used for Gram-negative isolates were: Ceftazidime (CAZ) $30 \mathrm{mcg}$, Cefipime (CPM) $30 \mathrm{mcg}$, Imipenem (IMP) $10 \mathrm{mcg}$, Co-Trimoxazole (COT) $1.25 / 23.75 \mathrm{mcg}$, Amikacin (AK) $30 \mathrm{mcg}$, Gentamicin (GEN) $10 \mathrm{mcg}$, Ciprofloxacin (CIP) $5 \mathrm{mcg}$, Ticarcillin/Clavulanic Acid (TCC) 75/10mcg, and Meropenem (MRP) 10mcg [12].

\section{RESULTS}

\subsection{Microbiological finding:}

From the total of 500 positive samples, the most common bacteria found was gram-positive bacteria; MRSA 215 (43\%), followed by gram-negative; $P$. aeruginosa and Acinetobacter baumannii (A. baumannii) $95(19 \%), 85(17 \%)$ respectively. Other organism included Non MRSA S. aureus, Kliebsella pneumoniae (K. pneumonia), Enterobacter cloacae (E. cloacae), Escherichia coli (E. coli), and Staphylococcus epidermidis( S. epidermidis), 50 (10\%), 25 (5\%), 39 (5.2\%), 17 (2.3\%), $11(2.2 \%), 8(1.1 \%), 7(1.4 \%)$ and 12 (2\%) respectively. (Table1)

Table1. Distribution types of bacteria

\begin{tabular}{|c|c|c|}
\hline Types of bacteria & Number & $\%$ \\
\hline \multicolumn{3}{|l|}{ Gram-positive } \\
\hline$M R S A$ & 215 & 43 \\
\hline S. aureus & 50 & 10 \\
\hline S. epidermidis & 12 & 2 \\
\hline Total & 277 & 55.4 \\
\hline \multicolumn{3}{|l|}{ Gram-negative } \\
\hline A. baumannii & 95 & 19 \\
\hline P. aeruginosa & 85 & 17 \\
\hline K. pneumoniae & 25 & 5 \\
\hline E. cloacae & 11 & 2.2 \\
\hline E. coli & 7 & 1.4 \\
\hline Total & 223 & 44.6 \\
\hline
\end{tabular}

\subsection{Antibiotic sensitivity:}

\subsubsection{Gram-positive bacteria:}

For MRSA, The highest resistances were seen against Co-Trimoxazole, Amikacin, Gentamicin, and Amoxicillin/Clavulanic Acid accounted; 97.1\%, 95.6\%, $91.3 \%$, and $82.3 \%$ respectively (Figure 1). But most antibiotics were active against non MRSA (S. aureus) and the resistant rates ranged from $0-17.6 \%$ (Figure 2).

In addition, $S$. epidermidis was full resistant to Amoxicillin/Clavulanic Acid 100\%, while the resistant rates were decreased for the other drugs (Figure 3). Also all gram-positive bacteria were highly sensitive for Vancomycin followed by Teicoplanin. (Figure1,2,3).

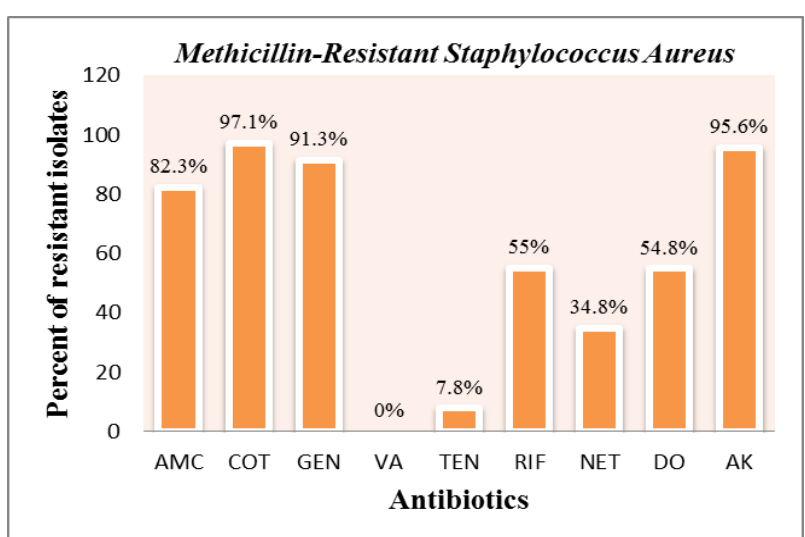

Figure1. Antibiotic resistance for MethicillinResistant Staphylococcus Aureus.

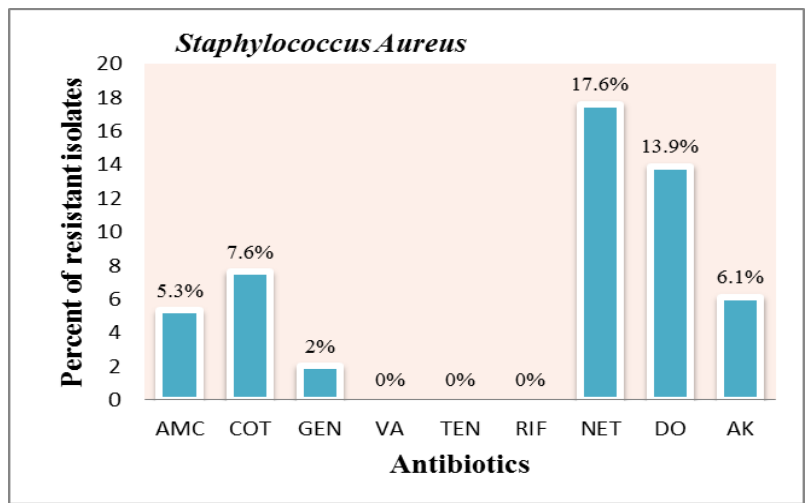

Figure2. Antibiotic resistance for Staphylococcus Aureus.

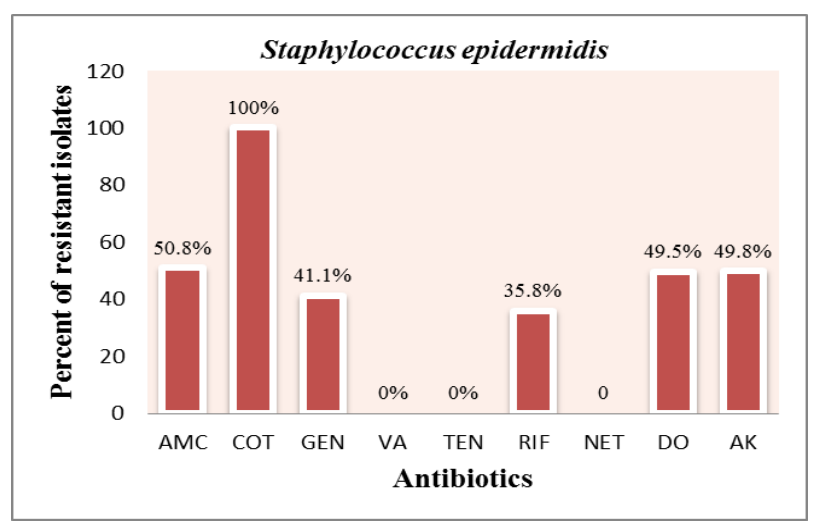

Figure3. Antibiotic resistance for Staphylococcus epidermidis. 


\subsubsection{Gram-negative bacteria:}

The resistant rate of $A$. baumannii was more than $80 \%$ for Ticarcillin/Clavulanic Acid, Ceftazidime, Cefipime, Amikacin and Gentamicin, but the resistant rates only for Imipenem was less than 30\% (Figure 4). In addition, the antibacterial drugs with drug resistant rate to $P$. aeruginosa more than $80 \%$ include only CoTrimoxazole, also resistant rate for Imipenem was lower than $30 \%$ and accounted $16.1 \%$ (Figure 5).

About $K$. pneumoniae drugs with drug resistant rate higher than $80 \%$ includes; Gentamicin, Ticarcillin/Clavulanic Acid, Ceftazidime and Gentamicin ranged from $80 \%$ to $93 \%$, while those with lowest drug resistant rate includes; Meropenem and Imipenem 7\% and 1.1\% (Figure 6).

The highest antibacterial resist rates for E. cloacae were Ticarcillin/Clavulanic Acid $80.1 \%$ and both Ceftazidime, Co-Trimoxazole 92.2\%; however there are lowest resistant rates for Meropenem and Imipenem $2.9 \%$ and $0.0 \%$ (Figure 7). Also for E. coli Meropenem and Imipenem were the active drugs with no resistant rates (Figure 8).

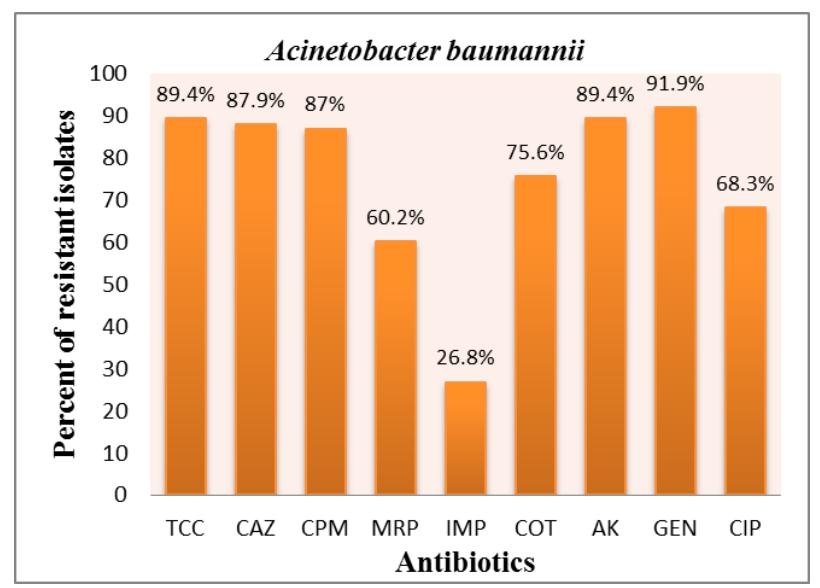

Figure4. Antibiotic resistance for Acinetobacter baumannii.

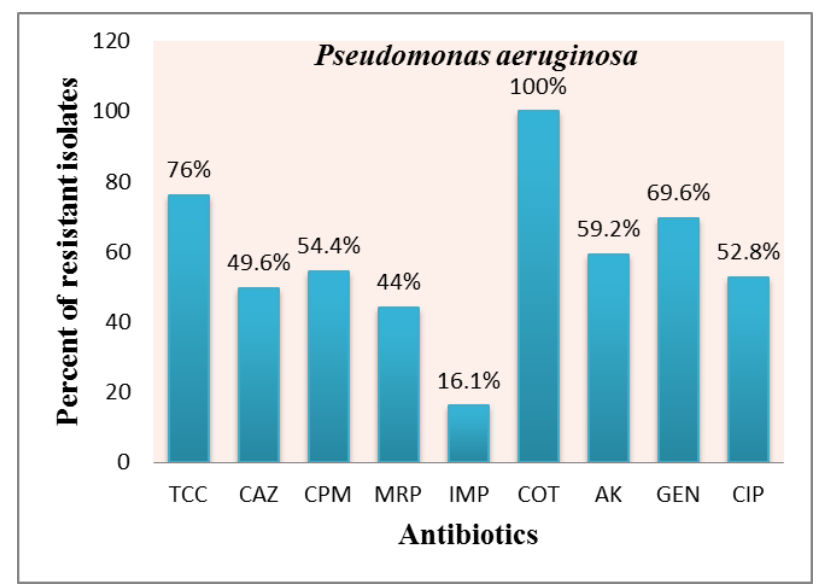

Figure5. Antibiotic resistance for Pseudomonas aeruginosa.

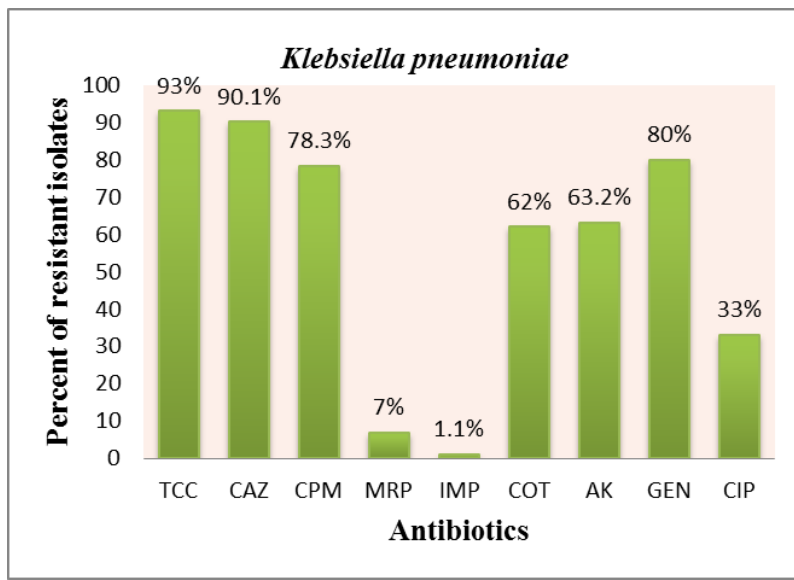

Figure6. Antibiotic resistance for Klebsiella pneumoniae.

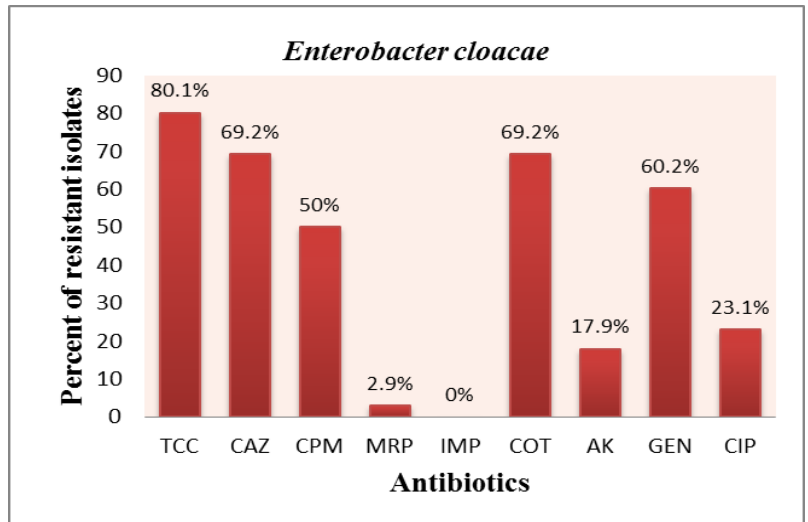

Figure7. Antibiotic resistance for Enterobacter cloacae.

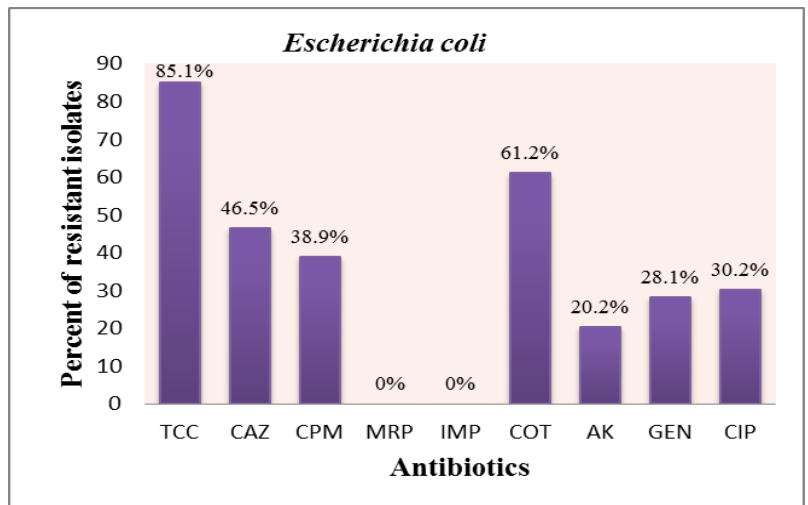

Figure8. Antibiotic resistance for Escherichia coli.

\subsection{Bacteriological infections:}

\section{DISCUSSION}

A total of 500 positive samples, we found that the highest proportion of positive samples were grampositive bacteria $55.4 \%$ followed by gram-negative bacteria $44.6 \%$ (Table 1). Our result in accordance with the previous study in China showed that the commonest pathogens were the gram-positive cocci [13]. But study conducted by Mayhall, C.G. in USA [14], and a study done by Balaban, I., et al in Turkey shows that the commonest pathogens were gram-negative bacteria [15].

This finding has several interpretations; first, grampositive organisms are major determinant of morbidity [16]. Second, endogenous gram-positive bacteria come 
from the patient's surface flora and rapidly colonize the burn wound surface after injury. On the other hand, delaying burn wound excision increases bacterial load, especially gram-positive and supports the rationale of early burn wound excision. Surgical excision decreases an average of 102 organisms per gram of tissue in both the early and delayed excision groups for gram-negative infection this is mentioned by Barret, J.P. and D.N. Herndon [17]. Burn wound surfaces are sterile after thermal injury; these wounds at the end become colonized with microorganisms. The nature and extent of the thermal injury along with the types and amounts of microbes colonize, and then gram-positive bacteria that survive the thermal insult, especially Staphylococci located deep within sweat glands and hair follicles, so the wound become colonized within the first 48 hours $[18,19]$.

In our study a total of 748 positive samples, among gram-positive infections; the first common isolated bacteria were MRSA $34.2 \%$ (Table 1). More recently, a 20- years review of the changes in bacterial isolates from burn wounds and their antibiograms in Europe showed that MRSA remain the most frequent gram-positive pathogen [20]. But this finding is disagree with the study conducted by Habibullah, S. which showed that the most common pathogens were Non MRSA (S. aureus) [16]. The prevalence of MRSA is various in clinical samples of burn patients as the result of distribution of PantonValentine Leukocidin (PVL) gene of the bacteria, Staphylococcal Cassette Chromosome mec (SCCmec) types and antibiotic resistant genes of the $M R S A$ isolates. The disruption of the normal skin barrier and the immune compromised status make burn an easy target for MRSA colonization [21]. Additionally, using various types of penicillin too much without changing and self uses of antibiotic randomly are risks for development of MRSA colonization in which supported by Branski, L.K., et al [22]. On the other hand, grampositive bacterial infection, especially MRSA comes from the patient's endogenous skin flora or the external environment predominantly colonizes the burn wounds [22].

We obtained that; A. baumannii is the second common causes of infection that accounted 19\% (Table 1). A study by Behzadnia, S., et al is agree with this result [6]. But a study by Wang, L.F., and his coauthors found that $A$. baumannii is the most common bacterial cause infection in burn patients [23]. The factor which may be lead to the presence of much Acinetobacter on a normal skin commensal and upper respiratory airway and it spreads easily [24].

\subsection{Antibacterial sensitivity isolates:}

All over the world, the resistant pattern for antibiotics in burn patients has been showing dramatic changes. Therefore, very important to identify the common pathogens which infected burns and their sensitivity to antibiotic for effective treatment of pediatric burn patients.

According to gram-positives pathogens, MRSA resistant rate is high for most of the antibiotics. The resistant rate was over $80 \%$ to Co-Trimoxazole,
Amikacin, Gentamicin, and Amoxicillin/Clavulanic Acid. A study from Iran showed the same result for Amoxicillin and Oxacillin, but it is differ for Gentamicin accounted $44.44 \%$ [25]. Another study has shown that MRSA antibiotic resistant rate for Amoxicillin and Oxacillin was $43.0 \%, 46.8 \%$ respectively [26]. The irrational use of these antibiotics may causes high resistant.

In our study, we found that all gram-positive bacteria have no resistance rate to Vancomycin followed by Teicoplanin $7.8 \%$ (figyre1,2,3). This finding is consistent with many other studies in burn wards $[6,16$, 25, 27].

Regarding to the gram-negative resistant isolates, the bacterial drug resistant rate to A. baumannii is high for Ticarcillin/Clavulanic Acid, Ceftazidime, Cefipime, Amikacin and Gentamicin more than $80 \%$ (Figure 4). Similar result was found by Rezai, M.S. and S. Shahmohammadi [28]. Also our study revealed that the less resistant antibiotic for A. baumannii was Imipenem only $26.8 \%$. This is similar to 37 centers in 11 European countries [29]. But a study by Bayram, Y., et al reported that $A$. baumannii resistant rate to Imipenem is high accounted $86 \%$ [30]. A previous study by Wang, L.F., and his coauthors demonstrated that the drug resistant to A. baumannii due to easily produce drug resistance by combining plasmid. A variety of resistant plasmids coexist, such as plasmid-mediated TEM-1 and TEM-2, TEM-2 $\beta$-lactamase, and chromosome-mediated $\beta$ lactamase; the change in penicillin-binding proteins and the permeability decrease of outer membrane proteins and also result drug resistance [23].

$P$. aeruginosa isolated from clinical samples in our study have shown differences, but it is still at high level of resistant to Co-Trimixasole only $100 \%$, but Imipenem was effective drug against $P$. aeruginosa with lees resistant rates (Figure 5). This finding is nearly in accordance to the previous study which is done by Souli, M., I. et al in Europe [29]. A study by Jafar, E., M.R. Shakibaie, and L. Poormasoomi mentioned that $P$. aeruginosa has different resistant rates because of the future plasmid different genes encoded, high stability, and low curing efficiency and easy transferred through conjugation to the other nosocomial pathogens [31].

For $K$. pneumoniae, the resistant rate was higher than $80 \%$ for Gentamicin, Ticarcillin/Clavulanic Acid, Ceftazidime and Gentamicin. But the most effective drugs were Meropenem and Imipenem with the resistant rates $7 \%$ and $1.1 \%$ (Figure 6). This finding is in consistent to results from South East Asian and with European Region [32]. Meropenem and Imipenem in our study were two active drugs without resistant rates for $E$. coli (Figure 8). This result is nearly in accordance to the previous studies [16, 27, 33]. But a study by Behzadnia, $\mathrm{S}$., et al showed that Imipenem was the highest drug resistant rate for E. coli [6].

Repeated using of above antibiotics could be factors lead to raises of resistant rates for most of them and cause incapability drugs activation. Because of the above finding and interpretations our Sulaimani health 
directorate must try to take high precaution about them and provide good antibiotics for their eradication.

\section{CONCLUSION}

Overall, gram-positive bacteria were the commonest pathogens followed by gram-negative. MRSA was the commonest frequent gram-positive bacteria detected and it is the major cause for infection. Vancomycin is an active drug against gram-positive bacteria and has no resistant rate to Staphylococcus species to the date of preparation of this study. The commonly isolated gramnegative bacteria showed increase resistant to the most of antibiotics except Imipenem is the less resistant. Regular microbial surveillance of burn patient should be done, also the pattern of isolation and drug resistant may help physician to implement adequate empirical antibiotic therapy and reducing emerging bacterial infection and their drug resistant.

\section{ACKNOWLEDGMENTS}

We gratefully thank the management of the Burns Centre Shaxawan Saeb Beg and his staff (Kamaran Amin Faraj, Chia Kamil Noori, Atta Huseen Ahmed, and Mokhtar Hussein Abdullah) for their excellent technical assistance in data collection.

\section{REFERENCE}

[1] M. Saaiq, S. Ahmad, and M. S. Zaib, "Burn wound infections and antibiotic susceptibility patterns at pakistan institute of medical sciences, islamabad, pakistan," World J Plast Surg, vol. 4, pp. 9-15, Jan 2015.

[2] D. Church, S. Elsayed, O. Reid, B. Winston, and R. Lindsay, "Burn wound infections," Clin Microbiol Rev, vol. 19, pp. 403-34, Apr 2006.

[3] G. Persson and J. Lindén, "Paediatric Burns and its Related Infections: a Qualitative Study Emphasizing the Preventive Work Conducted by Nurses in Dar es Salaam.," 2015.

[4] E. E. DiMuzio, D. P. Healy, P. Durkee, A. N. Neely, and R. J. Kagan, "Trends in bacterial wound isolates and antimicrobial susceptibility in a pediatric burn hospital," J Burn Care Res, vol. 35, pp. e304-11, Sep-Oct 2014.

[5] L. A. Barajas-Nava, J. Lopez-Alcalde, M. Roque i Figuls, I. Sola, and X. Bonfill Cosp, "Antibiotic prophylaxis for preventing burn wound infection," Cochrane Database Syst Rev, p. CD008738, 2013.

[6] S. Behzadnia, A. Davoudi, M. S. Rezai, and F. Ahangarkani, "Nosocomial infections in pediatric population and antibiotic resistance of the causative organisms in north of iran," Iran Red Crescent Med J, vol. 16, p. e14562, Feb 2014.

[7] G. M. AbdulRazzaq, "Pattern of antibiotic sensitivity and resistance pf uropathogenes among pediatric patients with urinary tract infection," Iraq J Pharm, vol. 1, p. 13, 2012.

[8] A. Zbuchea, O. Racasan, V. Falca, C. Mitache, and C. Vladescu, "Epidemiologic and Bacteriologic Study of the Burned Patients from the Plastic Surgery Department of the
County Emergency Hospital of Ploiesti, over a 4 Years Period (2010-2013)," Chirurgia (Bucur), vol. 110, pp. 362-7, Jul-Aug 2015.

M. Babakir-Mina, K. J. Rashid, S. S. Beg, C. K. Noori, M. I. M. Gubari, F. A. Mohialdeen, et al., "Epidemiological characteristics and antibiotic resistance of Acinetobacter baumannii isolated from burn patients," kjar, vol. 1, 2016.

[10] E. Salehifar, G. Khorasani, and S. Ala, "Timerelated Concordance Between Swab and Biopsy Samples in the Microbiological Assessment of Burn Wounds," Wounds, vol. 21, pp. 84-8, Mar 2009.

[11] A. Pechorsky, Y. Nitzan, and T. Lazarovitch, "Identification of pathogenic bacteria in blood cultures: comparison between conventional and PCR methods," J Microbiol Methods, vol. 78, pp. 325-30, Sep 2009.

[12] V. J., V. J., E. K., R. P., P. P., and H. C., "Basic laboratory procedures in clinical bacteriology, 2nd edition," WHO Geneva, pp. 91-93, 2003.

[13] D. Kun-qi, Y. Li-juan, and C. Qun-ying, "Investigation of Bacterial Species Distribution and Drug Resistance of Children With Wound Infection," Chinese Journal of Disinfection, vol. 5, p. 446, 2012.

[14] C. G. Mayhall, "The epidemiology of burn wound infections: then and now," Clin Infect Dis, vol. 37, pp. 543-50, Aug 152003.

[15] I. Balaban, G. Tanir, O. Metin Timur, F. N. Oz, T. Aydin Teke, G. I. Bayhan, et al., "Nosocomial infections in the general pediatric wards of a hospital in Turkey," Jpn J Infect Dis, vol. 65, pp. 318-21, Jul 2012.

[16] S. H, "Bacterial Profile of Paediatric Burn Wounds and their Antibacterial Sensitivity Patterns," Journal of Surgery: Open Access ( ISSN 2470-0991 ), vol. 2, 2016.

[17] J. P. Barret and D. N. Herndon, "Effects of burn wound excision on bacterial colonization and invasion," Plast Reconstr Surg, vol. 111, pp. 744-50; discussion 751-2, Feb 2003.

[18] U. Altoparlak, S. Erol, M. N. Akcay, F. Celebi, and A. Kadanali, "The time-related changes of antimicrobial resistance patterns and predominant bacterial profiles of burn wounds and body flora of burned patients," Burns, vol. 30, pp. 660-4, Nov 2004.

[19] S. Erol, U. Altoparlak, M. N. Akcay, F. Celebi, and M. Parlak, "Changes of microbial flora and wound colonization in burned patients," Burns, vol. 30, pp. 357-61, Jun 2004.

[20] M. Guggenheim, R. Zbinden, A. E. Handschin, A. Gohritz, M. A. Altintas, and P. Giovanoli, "Changes in bacterial isolates from burn wounds and their antibiograms: a 20-year study (1986-2005)," Burns, vol. 35, pp. 553-60, Jun 2009.

[21] O. Ergun, A. Celik, G. Ergun, and G. Ozok, "Prophylactic antibiotic use in pediatric burn units," Eur J Pediatr Surg, vol. 14, pp. 422-6, Dec 2004. 
[22] L. K. Branski, A. Al-Mousawi, H. Rivero, M. G. Jeschke, A. P. Sanford, and D. N. Herndon, "Emerging infections in burns," Surg Infect (Larchmt), vol. 10, pp. 389-97, Oct 2009.

[23] L. F. Wang, J. L. Li, W. H. Ma, and J. Y. Li, "Drug resistance analysis of bacterial strains isolated from burn patients," Genet Mol Res, vol. 13, pp. 9727-34, 2014.

[24] G. A. Tayh, "Risk Factors and Antimicrobial Resistance of Pathogens Isolated from Burn Units at Local Hospitals in Gaza Strip, Palestine," Islamic University-Gaza, Islamic University-Gaza, 2011.

[25] B. Dormanesh, S. Siroosbakhat, E. Khodaverdi Darian, and L. Afsharkhas, "MethicillinResistant Staphylococcus aureus Isolated From Various Types of Hospital Infections in Pediatrics: Panton-Valentine Leukocidin, Staphylococcal Chromosomal Cassette mec SCCmec Phenotypes and Antibiotic Resistance Properties," Jundishapur J Microbiol, vol. 8, p. e11341, Nov 2015.

[26] L. K. Elamenya, "Antimicrobial susceptibility of bacteria that cause wound sepsis in the paediatric surgical patients at Kenyatta national hospital," University of Nairobi, 2015.

[27] M. Imran, M. Faheem, V. Aslam, A. Hakeem, and A. Shah, "Wound infections and culture sensitivity pattern in pediatric burn patients," Journal of Postgraduate Medical Institute (Peshawar-Pakistan), vol. 23, 2011.
[28] M. S. Rezai and S. Shahmohammadi, "Nosocomial Infections in Iranian Pediatric Patients With Burn Injuries: A Review," Journal of Pediatrics Review, vol. 3, p. e680, 2015.

[29] M. Souli, I. Galani, and H. Giamarellou, "Emergence of extensively drug-resistant and pandrug-resistant Gram-negative bacilli in Europe," Euro Surveill, vol. 13, Nov 202008.

[30] Y. Bayram, M. Parlak, C. Aypak, and I. Bayram, "Three-year review of bacteriological profile and antibiogram of burn wound isolates in Van, Turkey," Int J Med Sci, vol. 10, pp. 1923, 2013.

[31] E. Jafar, M. R. Shakibaie, and L. Poormasoomi, "Isolation of a novel antibiotic resistance plasmid DNA from hospital isolates of Pseudomonas aeruginosa," Journal of Clinical \& Experimental Pathology, vol. 2013, 2013.

[32] WHO, "Antimicrobial resistance: global report on surveillance 2014," Webcast of Q\&A Session on global report on surveillance, p. 257 , 2014.

[33] B. Diler, N. Dalgic, C. A. Karadag, and A. I. Dokucu, "Epidemiology and Infections in a Pediatric Burn Unit: Experience of Three Years," Çocuk Enfeksiyon Dergisi/Journal of Pediatric Infection, vol. 6, pp. 40-45, 2012. 